\title{
Etude comparée de la tolérance au sel de diffé- rents mils : croissance, activités phosphoénol- pyruvate carboxylase et enzyme malique à NADP
}

\author{
Saliou BOURAÏMA, Danièle LAVERGNE \& Marie-Louise CHAMPIGNY \\ Université Paris-Sud, Photosynthèse et Métabolisme, Bâtiment 430, F 91405 Orsay Cedex
}

L'évaluation de la tolérance à $\mathrm{NaCl}$ a porté sur la production de matière fraîche, la teneur en protéines solubles, les activités phosphoénolpyruvate carboxylase et enzyme malique à NADP des organes aériens de Pennisetum typhoides L. cv. "Droô Zarzis », originaire de zones tunisiennes à terrains riches en sel, et pour comparaison, de $P$. americanum (L.) Leeke cv $23 \mathrm{DB}, P$. violaceum (L.) Rich. et $P$. mollissimum Hochst, originaires de zones tropicales semi-arides. Les traitements consistent en l'addition de $\mathrm{NaCl} 25 \mathrm{mM}$ ou $75 \mathrm{mM}$ au milieu de culture des plantes de $8 \mathrm{j}$. Les analyses portent sur les plantes de $26 \mathrm{j}$. La croissance exprimée par la production de matière fraîche permet de classer les 4 mils selon leur tolérance dégressive à $\mathrm{NaCl}: P$. typhoides qui montre une tendance marquée à l'halophilie, puis $P$. mollissimum, $P$. americanum $23 \mathrm{DB}$ et enfin $P$. violaceum. Une classification intégrant l'ensemble des résultats a été tentée. Elle précise le caractère très tolérant de $P$. typhoides et très sensible de $P$. violaceum. Les relations entre les capacités enzymatiques étudiées et le choc salin se traduisent par un effet positif sur la capacité phosphoénolpyruvate carboxylase des 4 mils mais de plus grande ampleur chez $P$. typhoides le plus tolérant, un effet positif sur la capacité enzyme malique à NADP de $P$. typhoides, mais négatif sur la capacité enzyme malique à NADP de $P$. violaceum, mil le moins tolérant. En $\mathbf{l}^{\mathrm{re}}$ approximation, sur le plan de l'assimilation de $\mathrm{CO}_{2}$, la tolérance à $\mathrm{NaCl}$ observée chez $P$. typhoides correspond donc à une stimulation de la capacité de fixation de $\mathrm{CO}_{2}$, associée à une production activee de $\mathrm{CO}_{2}$ à partir du malate dans les chloroplastes de cellules de gaine. La sensibilité à $\mathrm{NaCl}$ chez $P$. violaceum, mil non tolèrant, apparaît lice à un déficit de décarboxylation du malate dans les gaines. Il a été vérifié que $\mathrm{NaCl}$ ne modifie pas l'affinité de ces 2 enzymes pour leurs substrats, ni les sites antigéniques.

Mots clés additionnels : Pennisetum, protéines.

For the evaluation of salt tolerance, the fresh matter production, soluble protein content, phosphoenolpyrurate carboxylase and NADP-malic enzyme activities were analysed in the shoots of $P$. typhoides L. cv. 'Droô Zarzis', widespread in salt-rich soils in Tunisia and, for comparison, $P$. americanum (L.) Leeke cv $23 \mathrm{DB}, P$. violaceum (L.) Rich. and P. mollissimum Hochst originating from semiarid climates. Salt treatments consisted of addition of $\mathrm{NaCl}$ at $25 \mathrm{mM}$ or $75 \mathrm{mM}$ to the nutrient solution when seedlings were 8 days old. Seedlings were sampled for analysis at the age of 26 days. Classification of the four millets in the order of decreasing salt tolerance, estimated as fresh matter production, was as follows : $P$. typhoides, inclined to halophily, $P$. mollissimum, $P$. americanum $23 \mathrm{DB}$ and last, $P$. violaceum. Classification based on all criteria tested in this study confirmed $P$. typhoides as fairly NaCl-tolerant and $P$. violaceum as very sensitive. Salt stress brought about a positive effect on phosphoenolpyruvate carboxylase capacity in all four millets with a strong effect on the enzyme of the most tolerant $P$. typhoides, a positive effect on $P$. typhoides NADP-malic enzyme but a negative effect on NADP-malic enzyme of the less tolerant $P$. violaceum. These results were interpreted as suggesting that, relative to $\mathrm{CO}_{2}$ assimilation, the salt tolerance of $P$. typhoides could be explained by the stimulation of $\mathrm{CO}_{2}$ fixation associated with stimulation of $\mathrm{CO}_{2}$ release from malate in the bundle-sheath chloroplasts. $\mathrm{NaCl}$ sensitivity of the non-tolerant $P$. violaceum could be partly explained by a shortage of malate decarboxylase in the bundle-sheath chloroplasts. $\mathrm{NaCl}$ was found to affect neither the affinity of either enzyme for its substrate, nor its antigenic site.

Additional key words : Pennisetum, proteins. 


\section{ABRÉVIATIONS UTILISÉES :}

DTT : dithiothreitol ; EDTA : éthylène diamine tétra-acétate disodique ; MDH : malate déshydrogénase; MF : matière fraîche ; NADP-EM : enzyme malique à NADP ; PEP : phosphoénolpyruvate ; PEPC : phosphoénolpyruvate carboxylase.

\section{INTRODUCTION}

La section Penicillaria du genre Pennisetum comprend un grand nombre d'espèces et de variétés représentées par des formes spontanées et des formes cultivées. La diversité des écotypes est illustrée par le fait que si, en Afrique, la culture du mil est surtout pratiquée en zone sahélo-soudanaise, caractérisée par un climat tropical semi-aride, un mil céréalier, $P$. typhoides Droô Zarzis, est cependant cultivé en Tunisie dans des zones irriguées par l'eau de la Medjerba dont la teneur en $\mathrm{NaCl}$ est proche de $3 \mathrm{~g} / \mathrm{l}$.

Dans cette étude, nous avons cherché à mettre en évidence des caractères de résistance au sel de $P$. typhoides L. cv. "Droô Zarzis ", en cultivant les plantes dans des conditions contrôlées de salinité et en comparant leur comportement à celui de 3 Pennisetum d'origine géographique différente $P$. americanum (L.) Leeke $23 \mathrm{DB}$, forme cultivée sélectionnée en Géorgie (USA) et 2 formes sauvages, $P$. violaceum (L.) Rich. surtout localisé le long du fleuve Sénégal et $P$. mollissimum Hochst rencontré au Mali, en dehors des cultures de mil à chandelle. Ces mils ont tous un métabolisme carboné de type $\mathrm{C}_{4}$, à enzyme malique à NADP dominante (LAVERGNE et al., 1979).

L'évaluation de la résistance des plantes à $\mathrm{NaCl}$ a porté sur un critère de croissance, la quantité de matière fraîche des organes aériens, sur la teneur en protéines solubles en tant qu'expression du contenu enzymatique global et sur les activités phosphoénolpyruvate carboxylase (PEPC, EC 4.1.1.31) et enzyme malique à NADP (NADP-EM, EC 1.1.1.40) de plantes de $26 \mathrm{j}$ cultivées dans des milieux de salinité contrôlée. La comparaison de ces 2 enzymes chez les 4 mils a été également conduite par approche immunologique.

Le choix de l'étude de la PEPC et de la NADP-EM a été dicté par plusieurs considérations. La PEPC est responsable de la fixation initiale de $\mathrm{CO}_{2}$ dans les cellules de mésophylle des plantes de type $\mathrm{C}_{4}$ (HATCH \& SLACK, 1970). Couplée à l'enzyme malique à NADP, elle participe à l'homéostasie du $\mathrm{pH}$ intracellulaire (DAVIES, 1979 ; GUERN et al., 1983). La NADP-EM, localisée dans les chloroplastes des gaines périvasculaires catalyse la décarboxylation du malate et réalise ainsi l'approvisionnement en $\mathrm{CO}_{2}$ du cycle photosynthétique du carbone (cycle de Calvin-Benson). Elle est activement impliquée dans l'élimination de l'excès de malate chez les plantes de terrains salés (OSMOND \& LATIES, 1969). Des perturbations du contenu enzymatique des tissus végétaux sous l'effet de $\mathrm{NaCl}$ ont été signalées (OSMOND \& GREENWAY, 1972 ; GREENWAY \& OSMOND, 1972). Pour ce qui est de la PEPC et de la NADP-EM, de nombreuses études ont montré l'influence de la salinité ainsi que d'autres facteurs du milieu externe tels que l'éclairement ou la carence en phosphate sur leur niveau d'activité chez le maïs, le blé, le sorgho (HATCH, 1969 ; COLlins \& HAGUE, 1983 ; MiginiaC-MASLOW et al., 1983 ; Vidal \& GADAL, 1983). Le sel entraînerait chez Plantago maritima L. une telle exaltation de l'activité PEPC que le métabolisme carboné de cette plante en milieu salé a été comparé à un métabolisme de type $\mathrm{C}_{4}$ (FERRON et al., 1977). Chez Kalanchoe blossfeldiana, glycophyte présentant le métabolisme acide des Crassulacées (CAM), le traitement salin provoque une forte augmentation de la capacité de la PEPC (DIALlo \& QUEIROZ-ClARET, 1983). La NADP-EM des feuilles de Triglochin maritima L. est fortement inhibée par $\mathrm{NaCl}$ ou $\mathrm{KCl}$ (GREENWAY \& SIMS, 1974).

\section{MATÉRIEL ET MÉTHODES}

\section{A. Culture des plantes}

Les semences de $P$. typhoides, cv. "Droô Zarzis" et de $P$. mollisimum Hochst ont été fournies par le laboratoire de Génétique et Physiologie du Développement des Plantes du CNRS de Gif-sur-Yvette, France.

Les semences de $P$. violaceum (L.) Rich et de $P$. americanum synonyme de $P$. typhoides $\mathrm{L}$. ou de P. typhoideum (L.) Rich, cv. 23 DB ont été fournies par l'ORSTOM, Bondy, France.

Les semences sont mises directement à germer dans du sable humidifié à capacité de rétention $130 \mathrm{ml} / \mathrm{kg}$, à raison d'un grain par pot de 21 en salle conditionnée. L'éclairement, de 250-270 W. m-2 (mesure au quantum sensor) est fourni par les lampes OSRAM à décharge de $1000 \mathrm{~W}$. Le cycle photo-périodique est de $14 \mathrm{~h} / 10 \mathrm{~h}$ (jour/nuit). Les températures de jour et de nuit sont respectivement de 30 et $20^{\circ} \mathrm{C}$. L'humidité est d'environ 70 p. 100. L'arrosage est fait par l'eau déminéralisée jusqu'au stade 2 feuilles $(8 \mathrm{j}$ après le semis) où débute le traitement salin. Les plantes traitées sont alors arrosées par la solution de base contenant $\mathrm{NaCl}$ aux concentrations $25 \mathrm{mM}$ ou $75 \mathrm{mM}$. Les plantes témoins reçoivent la solution de base. Chaque lot comprend 3 plantes.

La solution de base type BLONDON (1968) a la composition suivante :

$\mathrm{KNO}_{3}: 2,7 \mathrm{mM} ; \mathrm{Ca}\left(\mathrm{NO}_{3}\right)_{2}: 4,5 \mathrm{mM} ; \mathrm{KH}_{2} \mathrm{PO}_{4}:$ $1 \mathrm{mM}$; $\left(\mathrm{NH}_{4}\right)_{2} \mathrm{SO}_{4}: 1 \mathrm{mM} ; \mathrm{MgSO}_{4}: 1,1 \mathrm{mM}$; $\mathrm{H}_{3} \mathrm{BO}_{3}: 0,05 \mathrm{mM} ; \mathrm{Na}_{2} \mathrm{MoO}_{4}: 0,014 \mathrm{mM} ; \mathrm{KCl}$ : $0,04 \mathrm{mM} ; \mathrm{CuSO}_{4}: 0,510^{-3} \mathrm{mM} ; \mathrm{MnSO}_{4}: 0,01 \mathrm{mM}$; $\mathrm{ZnSO}_{4}: 0,002 \mathrm{mM}$; EDTA ferrique : 0,1 mM. L'ion $\mathrm{Na}^{+}$n'est apporté que sous forme de traces par les impuretés des principaux sels utilisés. L'exigence d'un minimum de $\mathrm{Na}^{+}$dans le milieu de culture pour les plantes de type $\mathrm{C}_{4}$ est bien connue (BROWNELL \& CROSSLAND, 1972).

$\mathrm{Au} 26^{\mathrm{e}} \mathrm{j}$ de culture, les plantes sont récoltées 1 h 30 après le début de la photopériode. La totalité des organes aériens de chaque plante est pesée et fixée dans l'azote liquide. Trois cultures avec les traitements par $\mathrm{NaCl} 0,25 \mathrm{mM}, 75 \mathrm{mM}$ de $\mathrm{NaCl}$ ont été répétées. Les 3 cultures donnent des résultats qui évoluent toujours dans le même sens avec cependant une très grande variation des valeurs absolues d'une culture à l'autre, de telle sorte qu'on a été amené à pré- 
senter les résultats de la culture dont l'évolution est la plus représentative.

\section{B. Préparation des extraits enzymatiques}

Toutes les opérations ont été conduites à froid.

Les organes aériens préalablement fixés dans l'azote liquide sont broyés au mixer Polytron dans un tampon Tricine $50 \mathrm{mM}(\mathrm{pH} 8)$, DTT $5 \mathrm{mM}$ puis homogénéisés dans un broyeur de tissus Tenbroeck.

Le broyat est filtré sur nylon $(36 \mu \mathrm{m}$ de vide de maille). Le filtrat est centrifugé à $25000 \mathrm{~g}$ pendant $10 \mathrm{mn}$. Le surnageant obtenu constitue la fraction soluble utilisée pour la détermination des activités enzymatiques.

\section{Mesure des activités enzymatiques}

Les mesures sont effectuées à $30^{\circ} \mathrm{C}$.

L'activité PEPC est mesurée au spectrophotomètre Pye Unicam SP8 100 en suivant l'oxydation du $\mathrm{NADH}$ à $340 \mathrm{~nm}$ dans une réaction couplée avec la malate déshydrogénase (SIGMA). L'extrait enzymatique est incubé $5 \mathrm{mn}$ à $30^{\circ} \mathrm{C}$ dans le milieu réactionnel suivant: Tricine $50 \mathrm{mM}, \mathrm{pH} 8$, NADH $0,25 \mathrm{mM}$, DTT $5 \mathrm{mM}, \mathrm{MgCl}_{2} 10 \mathrm{mM}, \mathrm{NaHCO}_{3} 20 \mathrm{mM}, \mathrm{MDH}$ $0,02 \mathrm{U}$. La réaction est initiée par le PEP $5 \mathrm{mM}$ (LANE et al., 1969). Le volume réactionnel est de $1 \mathrm{ml}$.

L'activité NADP-EM est mesurée par spectrophotométrie en suivant la réduction du NADP, à $340 \mathrm{~nm}$. Le volume réactionnel total est de $1 \mathrm{ml}$ dans le tampon Tricine $50 \mathrm{mM}$, pH 8,3. Les substrats et cofacteurs sont: malate $5 \mathrm{mM}$, NADP $0,5 \mathrm{mM}$, EDTA $0,1 \mathrm{mM}, \mathrm{MgCl}_{2} 2 \mathrm{mM}$. Un essai sans $\mathrm{MgCl}_{2}$ est effectué dans chaque expérience afin de corriger une réduction possible du NADP due à l'activité malate deshydrogénase à NADP. La réaction est initiée par l'addition du malate $5 \mathrm{mM}$.

\section{Analyse immunologique}

Un volume de $10 \mathrm{ml}$ de préparation enzymatique est précipité par $\left(\mathrm{NH}_{4}\right)_{2} \mathrm{SO}_{4}, 7 \mathrm{H}_{2} \mathrm{O}$ entre 10 et 60 p. 100 de saturation. Le culot obtenu après $20 \mathrm{mn}$ de centrifugation, à $48000 \mathrm{~g}$ est repris dans $250 \mu \mathrm{l}$ de tampon Tricine $50 \mathrm{mM}$ pH 8,5. La méthode de double diffusion d'OUCHTERLONY (1958) est employée avec un anticorps de lapin contre la PEPC du sorgho (Sorghum vulgare) (VIDAL \& GADAL, 1983) et un anticorps de lapin contre la NADP-EM de sorgho (JOLIVET \& VIDAL, comm. pers.).

\section{E. Dosage des protéines solubles}

Les protéines sont dosées sur les extraits enzymatiques après précipitation à l'acide trichloracétique à 20 p. 100. Le précipité est repris par une solution de $\mathrm{NaOH} 1 \mathrm{M}$ et dosé par colorimétrie selon LOWRY et al. (1951). La quantité de protéines est déterminée par référence à une courbe d'étalonnage faite avec la sérum albumine bovine entre 0 et $250 \mu \mathrm{g}$ de protéines.

\section{RÉSULTATS}

\section{A. Croissance des plantes}

Au terme de $26 \mathrm{j}$ de culture, les plantes de $P$. violaceum, $P$. mollissimum et $P$. americanum cultivées sans traitement $\mathrm{NaCl}$ sont au stade 10-12 feuilles avec 3 talles à l'aisselle des feuilles 3-4. La feuille 1 est la $1^{\text {re }}$ feuille apparue. $P$. typhoides cv. "Droô Zarzis » présente 8-10 feuilles avec un seul talle à l'aisselle de la feuille 3. Par sa grande taille $P$. typhoides traité par $\mathrm{NaCl} 25 \mathrm{mM}$ se démarque nettement de son témoin et des 3 autres mils. Il présente 10-13 feuilles avec de nombreux talles. $P$. violaceum, $P$. mollissimum et $P$. americanum traités par $\mathrm{NaCl} 25 \mathrm{mM}$ sont très semblables à leurs témoins. Les plantes soumises au traitement $75 \mathrm{mM}$ de $\mathrm{NaCl}$ apparaissent rabougries (78 feuilles) par rapport au témoin cultivé en absence de sel pour $P$. violaceum, $P$. americanum et $P$. mollissimum tandis que la morphologie et la taille de $P$. typhoides se rapprochent de celles de son témoin.

\section{B. Quantité de matière fraîche et teneur en protéines solubles des organes aériens}

Pour les 4 mils témoins (cultures non traitées par $\mathrm{NaCl}$, on remarque que la production de matière fraîche en $26 \mathrm{j}$ est plus faible chez $P$. typhoides, plus forte chez $P$. violaceum que chez les 2 autres mils. Elle est affectée de façon différente par l'addition de $\mathrm{NaCl}$ dans le milieu de culture. La concentration $25 \mathrm{mM}$ favorise la croissance du « Droô Zarzis » qui tend à doubler. Elle ne modifie pas la quantité de matière fraîche de $P$. americanum « $23 \mathrm{DB} »$ et $P$. mollissimum. Elle diminue la production de matière fraîche de $P$. violaceum. A la concentration $75 \mathrm{mM}$, le sel entrave la production de matière fraîche des 4 mils et en particulier de $P$. violaceum (tabl. 1).

Chez les témoins, la teneur en protéines solubles exprimée en $\mathrm{g}$ de matière fraîche est sensiblement égale pour le cv. « $23 \mathrm{DB} », P$. violaceum et $P$. $\mathrm{mol}$ lissimum, alors qu'elle est plus élevée chez le «Droô Zarzis ». $\mathrm{NaCl}$ aux concentrations 25 et $75 \mathrm{mM}$ ne modifie pas ces teneurs (tabl. 1). Les variations des

\section{TABLEAU 1}

Influence de la concentration en $\mathrm{NaCl}$ du milieu de culture sur la production de matière fraîche et la teneur en protéines solubles des organes aériens de mils de 26 jours.

Effect of $\mathrm{NaCl}$ in the nutrient solution on the fresh weight and soluble protein content of the shoots of 26-day-old pearl millets.

\begin{tabular}{lccc}
\hline & $\begin{array}{c}\mathrm{NaCl} \\
\mathrm{mM}\end{array}$ & $\begin{array}{c}\mathrm{MF} \\
\mathrm{g} \text { par plante }\end{array}$ & $\begin{array}{c}\text { Protéines } \\
\text { solubles } \\
\mathrm{mg} \cdot \mathrm{g}^{-1} \mathrm{MF}\end{array}$ \\
\hline P. typhoides & 0 & 7,5 & 11,6 \\
cv. Drô̂ Zarzis & 25 & 18,3 & 7 \\
& 75 & 3,7 & 8,6 \\
P. americanum & 0 & 12 & 7 \\
cv. 23 DB & 25 & 10 & 7 \\
& 75 & 4,1 & 7,5 \\
$P$. violaceum & 0 & 23,7 & 5 \\
& 25 & 11,7 & 5,2 \\
P. mollissimum & 75 & 7 & 5,2 \\
& 0 & 12,2 & 8 \\
& 25 & 12,3 & 7,5 \\
& 75 & 7,4 & 8,4 \\
\hline
\end{tabular}


quantités de protéines solubles par plante suivent celles de la masse de matière fraîche.

L'augmentation de la production de matière fraîche sous l'effet de $\mathrm{NaCl} 25 \mathrm{mM}$ chez le "Droô Zarzis " montre une tendance marquée à l'halophilie chez ce mil. Il ressort de ces $1^{\text {res }}$ observations la possibilité d'un classement de 4 mils selon leur sensibilité dégressive vis-à-vis de $\mathrm{NaCl}: P$. typhoides cv. « Droô Zarzis », $P$. mollissimum, $P$. americanum cv. « $23 \mathrm{DB} »$, $P$. violaceum. Pour la suite des résultats, ce classement a été choisi afin d'en faciliter la lecture.

\section{Activité PEPC}

Qu'elle soit exprimée par $g$ de matière fraîche ou par $\mathrm{mg}$ de protéines solubles, l'activité PEPC ne présente pas de variation marquée entre les différents mils cultivés en absence de sel (tabl. 2). L'effet du traitement par $\mathrm{NaCl}$ en revanche se présente comme suit : chez le "Droô Zarzis ", l'activité par g de matière fraîche et par $\mathrm{mg}$ de protéines solubles est augmentée par le sel à la concentration $25 \mathrm{mM}$. Elle n'est pas affectée de façon interprétable chez $P$. mollissimum, $P$. americanum et $P$. violaceum par le sel à cette concentration. Mais la présence de $\mathrm{NaCl} 75 \mathrm{mM}$ dans le milieu de culture stimule l'activité PEPC par unité de matière fraîche et par $\mathrm{mg}$ de protéines solubles chez tous les mils étudiés. Puisque la quantité de protéines solubles totales par plante diminue en présence de $\mathrm{NaCl} 75 \mathrm{mM}$, ce résultat suggère que la PEPC est moins affectée par la salinité que l'ensemble des protéines solubles.

$\mathrm{Le} \mathrm{NaCl}$ aux concentrations étudiées ne modifie pas le $\mathrm{Km}$ apparent pour le PEPC de l'activité PEPC in vitro. Ce $\mathrm{Km}$ est $0,6,0,55,0,48,0,56$ respectivement pour $P$. typhoides cv. "Droô Zarzis ", P. mollissimum, $P$. americanum cv. " $23 \mathrm{DB} », P$. violaceum cultivés en présence ou en absence de sel. Le sel

TABLEAU 2

Influence de la concentration en $\mathrm{NaCl}$ du milieu de culture sur les activités PEPC et NADP-EM des organes aériens de mils de 26 jours

Effect of $\mathrm{NaCl}$ in the nutrient solution on the PEPC and NADP-EM activities of the shoots of 26-day-old pearl millet.

\begin{tabular}{|c|c|c|c|c|c|}
\hline & \multirow{2}{*}{$\begin{array}{c}\mathrm{NaCl} \\
\mathrm{mM}\end{array}$} & \multicolumn{2}{|c|}{$\begin{array}{c}\text { PEPC } \\
\mu \text { mol NADH oxydé } \\
\mathrm{mn}^{-1}\end{array}$} & \multicolumn{2}{|c|}{$\begin{array}{c}\text { NADP-EM } \\
\mu \mathrm{mol} \text { NADP réduit } \\
\mathrm{mn}^{-1}\end{array}$} \\
\hline & & $\mathrm{g}^{-1} \mathrm{MF}$ & $\begin{array}{l}\mathrm{mg}^{-1} \\
\text { protéines } \\
\text { solubles }\end{array}$ & $\mathrm{g}^{-1} \mathrm{MF}$ & $\begin{array}{l}\mathrm{mg}^{-1} \\
\text { protéines } \\
\text { solubles }\end{array}$ \\
\hline \multirow{3}{*}{$\begin{array}{l}\text { P. typhoides } \\
\text { cv. Droô Zarzis }\end{array}$} & 0 & 8,6 & 0,7 & 7 & 0,6 \\
\hline & 25 & 10,4 & 1,4 & 5,6 & 0,8 \\
\hline & 75 & 13 & 1,5 & 12 & 1,4 \\
\hline \multirow[t]{3}{*}{ P. mollissimum } & 0 & 10 & 1,2 & 7 & 0,9 \\
\hline & 25 & 11 & 1,4 & 7,2 & 1 \\
\hline & 75 & 14 & 1,6 & 6 & 0,7 \\
\hline \multirow{3}{*}{$\begin{array}{c}\text { P. americanum } \\
\text { cv. } 23 \mathrm{DB}\end{array}$} & 0 & 8 & 1,1 & 6 & 0,9 \\
\hline & 25 & 8 & 1,1 & 6 & 0,9 \\
\hline & 75 & 12 & 1,6 & 10 & 1,3 \\
\hline \multirow{3}{*}{ P. violaceum } & 0 & 10 & 1,5 & 3,6 & 0,7 \\
\hline & 25 & 11 & 1,5 & 3,1 & 0,6 \\
\hline & 75 & 14 & 2,2 & 3,4 & 0,6 \\
\hline
\end{tabular}

n'altère donc pas l'affinité de l'enzyme pour son substrat. Cependant, le traitement par $\mathrm{NaCl} 50 \mathrm{mM}$ et $75 \mathrm{mM}$ des extraits bruts de mils cultivés en absence de sel provoque une inhibition de l'activité in vitro mesurée en présence de PEP $1,5 \mathrm{mM}$. La concentration de PEP $3 \mathrm{mM}$ lève cette inhibition au moins partiellement, sinon totalement (tabl. 3). A ce point de vue, la PEPC de "Droô Zarzis " est beaucoup plus inhibée que celle des autres mils notamment de $P$. violaceum. Le traitement par $\mathrm{NaCl}$ des extraits bruts de plantes cultivées en présence de $\mathrm{NaCl} 75 \mathrm{mM}$ provoque aussi une inhibition de l'activité PEPC in vitro, mais l'inhibition n'est pas levée par le PEP (tabl. 4). Des effets similaires ont été signalés par GREENWAY \& OSMOND (1972) travaillant avec des extraits de feuilles d'Atriplex spongiosa, espèce connue pour sa résistance élevée à la salinité.

\section{TABLEAU 3}

Effet du prétraitement in vitro par $\mathrm{NaCl}$, d'extraits de mils cultivés en absence de $\mathrm{NaCl}$, sur l'activité PEPC. L'activité de l'extrait non prétraité par $\mathrm{NaCl}$ (témoin) est prise égale à 100. Deux expériences sont présentées (moyenne de 2 mesures par expérience).

Effect of in vitro pretreatment by $\mathrm{NaCl}$ of crude extracts from shoots of pearl millets grown without $\mathrm{NaCl}$, on PEPC activity. The activity of the non-preteated extract is taken as 100. Two experiments are shown (mean of two measurements per experiment).

\begin{tabular}{|c|c|c|c|c|c|}
\hline & \multirow{3}{*}{$\begin{array}{l}\text { Traitement } \\
\text { de l'extrait } \\
\mathrm{NaCl}, \mathrm{mM}\end{array}$} & \multicolumn{4}{|c|}{ Activite PEPC, $\%$ du témoin } \\
\hline & & \multicolumn{4}{|c|}{$\mathrm{PEP}$ in vitro, $\mathrm{mM}$} \\
\hline & & \multicolumn{2}{|c|}{1,5} & \multicolumn{2}{|c|}{3} \\
\hline \multirow{3}{*}{$\begin{array}{l}\text { P. typhoides } \\
\text { cv. Droô Zarzis }\end{array}$} & 25 & 95 & 93 & 99 & 100 \\
\hline & 50 & 58 & 61 & 75 & 78 \\
\hline & 75 & 45 & 39 & 74 & 72 \\
\hline \multirow[t]{3}{*}{ P. mollissimum } & 25 & 98 & 98 & 99 & 100 \\
\hline & 50 & 63 & 62 & 100 & 99 \\
\hline & 75 & 57 & 59 & 98 & 100 \\
\hline \multirow{3}{*}{$\begin{array}{l}\text { P. americanum } \\
\mathrm{cv} .23 \mathrm{DB}\end{array}$} & 25 & 98 & 97 & 99 & 99 \\
\hline & 50 & 70 & 73 & 99 & 100 \\
\hline & 75 & 58 & 65 & 92 & 91 \\
\hline \multirow[t]{3}{*}{ P. violaceum } & 25 & 98 & 98 & 100 & 100 \\
\hline & 50 & 75 & 74 & 98 & 100 \\
\hline & 75 & 60 & 64 & 98 & 99 \\
\hline
\end{tabular}

\section{Activité NADP-EM}

Sur le milieu sans sel, $P$. violaceum se distingue par une activité NADP-EM plus faible que celle des autres mils (tabl. 2). $\mathrm{NaCl} 25 \mathrm{mM}$ n'affecte pas sensiblement l'activité par unité de matière fraîche ou de protéines solubles. En revanche, à $75 \mathrm{mM}$, il la stimule chez le " Droô Zarzis » et chez $P$. americanum, que l'activité soit exprimée par $\mathrm{g}$ de matière fraîche ou par $\mathrm{mg}$ de protéines solubles.

Le $\mathrm{Km}$ apparent de la NADP-EM pour le malate est $0,57,0,50,0,35,0,47 \mathrm{mM}$ respectivement pour l'enzyme des extraits bruts de "Droô Zarzis ", $P$. mollissimum, $P$. americanum et $P$. violaceum. Ces $\mathrm{Km}$ ne sont pas affectés par la présence de $\mathrm{NaCl}$ dans le milieu de culture. Le traitement des extraits bruts des plantes témoins pour le $\mathrm{NaCl}$ in vitro ne modifie pas les activités NADP-EM. 


\section{E. Analyse immunologique}

Les tests d'immunologie effectués en présence des immun-sérums anti-PEPC et anti-NADP-EM de sorgho, sur les extraits enzymatiques des 4 mils culti- vés en présence ou en absence de $\mathrm{NaCl}$, révèlent une parfaite identité des PEPC, d'une part, des NADPEM, d'autre part, entre les 4 mils et selon les conditions de culture (fig. $1 \mathrm{~A}$ et $1 \mathrm{~B}$ ).

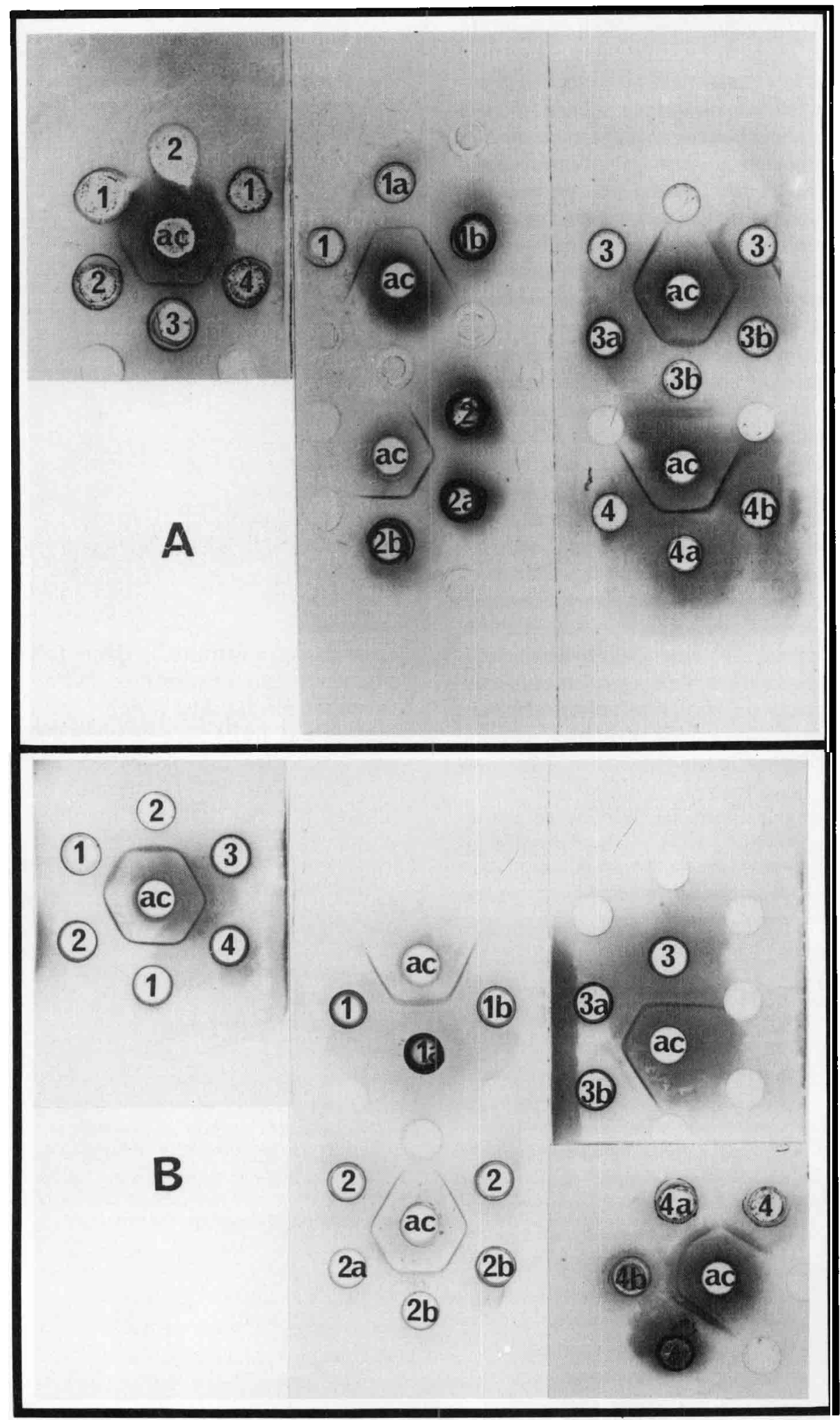

Figure 1

Analyse immunologique par le test de la double diffusion d'OuCH TERIONY (1958) de la PEPC (A), de la NADP-EM (B) de (I), P americanum (v. $23 \mathrm{DB} ;$ (2), P. typhoides ( 1 . Droô Larzis : (3), P mollissimum ; (4). P. violactum. Influence de l'addition de $\mathrm{NaCl}$ dans le milieu de cullure : $(1,2,3,4)$, temoins sans $\mathrm{NaCl} ;(\mathrm{la}, 2 a, 3 a$, 4a), $\mathrm{NaCl} 25 \mathrm{mM} ;(1 \mathrm{~b}, 2 \mathrm{~b}, 3 \mathrm{~b}, 4 \mathrm{~b}), \mathrm{NaCl} 75 \mathrm{mM}$. (ac), dans le puits central $10 \mathrm{\mu ml}$ d'antiserum de lapin contre la PEPC de sorgho (A), contre la NADP-EM de sorgho $(B)$. Les puits périphériques contiennent lo $\mu$ l des extraits protéiques des fenilles des mils.
Immunological analysis by the OUCHTERLONI double diffusion tes of $P E P C$ (A) and NADP-ME (B) from: 1) P. americanum $c v$. $23 D B$; 2) P. typhoïdes $c v$. Droô Zargis ; 3) P. mollissimum ; 4) P. violaceum : 1-4) controls without $\mathrm{NaCl}, \mathrm{la}-4 a) 25 \mathrm{mM} \mathrm{NaCl}$, lb-4b) $75 \mathrm{mM} \mathrm{NaCl}$. Centre well (ac) with $10 \mu$ rabbit antiserum against sorghum PEPC (A) or NADP-ME (B). Surrounding wells with $10 \mu$ protein extract from Pennisetum leaves. 


\section{DISCUSSION}

P. typhoides cv. "Droô Zarzis " se distingue très nettement des 3 autres mils étudiés par sa tendance à l'halophilie. Des différences variétales ont aussi été signalées chez le maïs (SIEGEL et al., 1980) et le lupin. En effet, un gain de 50 p. 100 de matière fraîche est provoqué chez Lupinus luteus mais non chez Lupinus angustifolius, par la culture sur milieu salé (VAN STEVENINK et al., 1982). P. americanum, $P$. mollissimum et $P$. violaceum soumis au traitement par $\mathrm{NaCl}$ $75 \mathrm{mM}$ présentent au niveau de leur morphologie et de leur croissance des caractéristiques déjà décrites chez les glycophytes sensibles au sel (atriplex, haricot) c'est-à-dire, réduction du nombre de feuilles et de leur croissance pondérale (HAMZA, 1977 ; MEIRI \& POLJAKOFF-MA YBER, 1970).

Les teneurs en protéines solubles ne présentent pas de variations significatives sous l'effet du sel chez les mils étudiés ce qui est comparable aux observations sur l'orge soumis à un traitement par $\mathrm{NaCl} 200 \mathrm{mM}$ (ASLAM et al., 1984). Cependant, une diminution des teneurs en protéines a été signalée chez le pois (Kahane \& PolJakoff-Mayber, 1968). Il est toutefois à noter que les différences ainsi observées peuvent ne dépendre que de l'âge physiologique des plantes et du moment d'application du traitement au sel (LEVITT, 1972). L'augmentation des activités PEPC et NADP-EM par unité de protéines solubles chez les plantes traitées par $\mathrm{NaCl} 75 \mathrm{mM}$ suggère un enrichissement relatif des pools de protéines solubles en ces 2 enzymes par rapport aux autres protéines, comme cela a déjà été signalé chez le blé (MIGINIAC-MASLOW et al., 1983).

L'augmentation de l'activité PEPC par le traitement des plantes par $\mathrm{NaCl} 75 \mathrm{mM}$ est une manifestation de la réponse des plantes au choc salin qui peut être comparée à l'augmentation de l'activité PEPC chez le maïs et chez le blé en réponse à des contraintes nutritionnelles (HAGUES \& SIM, 1980; MiginIACMASLOW et al., 1983). L'activité PEPC mesurée in
TABLEAU 4

Effet du prétraitement in vitro par $\mathrm{NaCl}$, d'extraits de mils cultivés en présence de $\mathrm{NaCl} 75 \mathrm{mM}$, sur l'activité PEPC. L'activité de l'extrait non prétraité par $\mathrm{NaCl}$ (témoin) est prise égale à 100. Deux expériences sont présentées (moyenne de 2 mesures par expérience).

Effects of in vitro pretreatment by $\mathrm{NaCl}$ of crude extracts from shoots of pearl millets grown on $75 \mathrm{mM} \mathrm{NaCl}$, on PEPC activity. The activity of the non-pretreated extracts is taken as 100. Two experiments are shown (mean of two measurements per experiment).

\begin{tabular}{|c|c|c|c|c|c|}
\hline & \multirow{3}{*}{$\begin{array}{l}\text { Traitement } \\
\text { de l'extrait } \\
\mathrm{NaCl}, \mathrm{mM}\end{array}$} & \multicolumn{4}{|c|}{ Activite PEPC, $\%$ du témoin } \\
\hline & & \multicolumn{4}{|c|}{$\mathrm{PEP}$ in vitro, $\mathrm{mM}$} \\
\hline & & \multicolumn{2}{|c|}{1,5} & \multicolumn{2}{|c|}{3} \\
\hline P. typhoides & 25 & 88 & 92 & 90 & 92 \\
\hline \multirow[t]{2}{*}{ Droô Zarzis } & 50 & 86 & 87 & 89 & 91 \\
\hline & 75 & 86 & 89 & 87 & 90 \\
\hline \multirow[t]{3}{*}{ P. mollissimum } & 25 & 87 & 85 & 89 & 87 \\
\hline & 50 & 86 & 85 & 90 & 87 \\
\hline & 75 & 89 & 90 & 89 & 91 \\
\hline \multirow{3}{*}{$\begin{array}{l}\text { P. americanum } \\
\text { cv. } 23 \mathrm{DB}\end{array}$} & 25 & 83 & 87 & 82 & 85 \\
\hline & 50 & 86 & 86 & 100 & 92 \\
\hline & 75 & 86 & 87 & 87 & 89 \\
\hline \multirow[t]{3}{*}{ P. violaceum } & 25 & 86 & 82 & 88 & 84 \\
\hline & 50 & 89 & 90 & 94 & 92 \\
\hline & 75 & 87 & 87 & 87 & 87 \\
\hline
\end{tabular}

vitro dans les extraits de tissus foliaires peut être considérée comme l'expression de l'activité potentielle ou capacité enzymatique. Le fait que l'activité PEPC soit inhibée par traitement in vitro des extraits de feuilles par $\mathrm{NaCl}$ alors que la capacité PEPC des tissus est soit non modifiée, soit stimulée par le traitement des plantes par $\mathrm{NaCl}$, laisse supposer l'existence in situ d'une protection de la PEPC vis-à-vis des ions $\mathrm{Na}^{+}$et $\mathrm{Cl}^{-}$. Cette protection serait meilleure chez «Droô Zarzis " que chez les autres cultivars, en particulier que chez $P$. violaceum.

L'absence de modification des sites antigéniques de la PEPC et de la NADP-EM par le traitement salin

TABLEAU 5

Tolérance à $\mathrm{NaCl}$ de P. typhoides $c v$ "Droô Zarzis", P. mollissimum, P. americanum, P. violaceum, calculée par la somme algébrique des effets des traitements par $\mathrm{NaCl} 25 \mathrm{mM}$ et $75 \mathrm{mM}$ sur la quantité de matière fraîche, la teneur en protéines solubles, les activités PEPC et NADP-EM. Les effets sont exprimés par la variation en p. 100 des mesures obtenues avec les plantes non traitees.

Sensitivity to $\mathrm{NaCl}$ of P. typhoides cv "Droo Zarzis", P. americanum, P. mollissimum, P. violaceum, calculated as the algebric sum of the effects of treatments by $25 \mathrm{mM}$ and $75 \mathrm{mM} \mathrm{NaCl}$ on fresh weight, soluble protein contem, PEPC' and NADP-EM activities. Effects are expressed by variation in of corresponding values for non-treated plants.

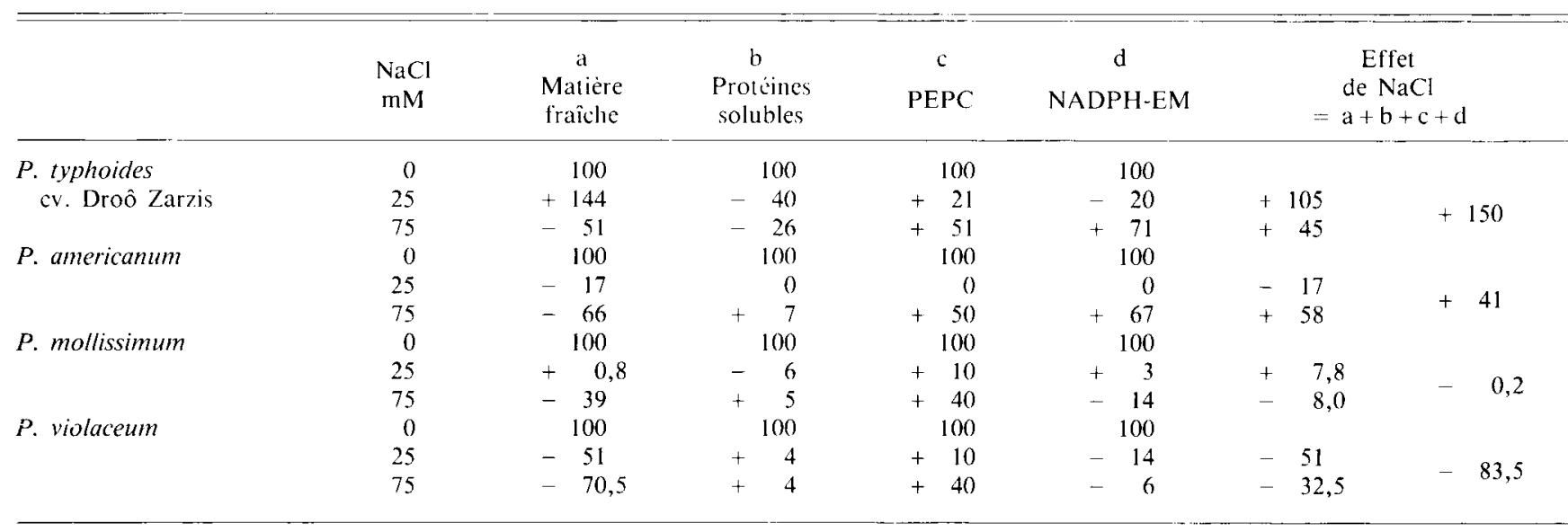


des plantes, et l'augmentation des activités enzymatiques correspondantes laissent supposer que les mécanismes mis en jeu par les mils en réponse au choc salin dépendent plus de la capacité des plantes à compartimenter $\mathrm{Na}^{+}$voire $\mathrm{Cl}^{-}$que de l'adaptabilité des systèmes enzymatiques (GREENWAY \& MUNNS, 1980).

Le rôle fondamental de la compartimentation intracellulaire des métabolites a été signalé par OSMOND \& LATIES (1969). Des mesures physiologiques concordent pour suggérer l'existence d'une expulsion active du sodium cytoplasmique vers l'apoplasme ou vers la vacuole, protégeant ainsi les équipements enzymatiques du cytoplasme, notamment dans les organes aériens (GREENWAY \& MUNNS, 1980).

Afin de comparer le comportement des 4 mils vis-àvis de la salinité du milieu de culture, une classification intégrant l'ensemble des résultats présentés dans cette étude a été tentée (tabl. 5). Les effets du sel sur la production de matière fraîche, la teneur en protéines solubles, les capacités PEPC et NADP-EM des organes aériens sont calculés par la variation des valeurs de chaque paramètre chez les plantes traitées, en p. 100 de celles des plantes témoins. La tolérance est traduite par une valeur positive et la sensibilité par une valeur négative. Cette approche confirme la classification présentée plus haut sur la base de la production de matière fraîche. Elle précise de plus le caractère très tolérant de $P$. tvphoides "Drô Zarzis" $(+150)$, mais très sensible de $P$. violaceum $(-83,5)$. $P$. americanum et $P$. mollisimum ont un comportement intermédiaire. Soit les mécanismes de réponse au choc salin, soit leur intensité sont différents chez les
4 mils, $P$. typhoides " Droô Zarzis " et $P$. violaceum représentent 2 comportements extrêmes qu'il serait intéressant d'utiliser comme sujet d'étude des modes d'adaptabilité de Pennisetum à $\mathrm{NaCl}$.

Les relations entre les capacités PEPC et NADPEM et le choc salin se traduisent par un effet positif sur la capacité PEPC quel que soit le comportement des plantes, un effet positif $(+51)$ sur la capacité NADP-EM de $P$. typhoides, mil tolérant, mais négatif $(-20)$ sur la capacité NADP-EM de $P$. violaceum, mil, très sensible. Ces 2 enzymes impliquées dans le métabolisme carboné des plantes de type $\mathrm{C}_{4}$ sont donc affectées différemment lors du traitement des plantes par $\mathrm{NaCl}$. En $1^{\text {re }}$ approche, la capacité NADP-EM apparaît être un marqueur de tolérance à $\mathrm{NaCl}$ chez le mil. En effet, la tolérance à $\mathrm{NaCl}$ observée che $P$. typhoides "Droô Zarzis" peut être considérée comme associée à une production activée de $\mathrm{CO}_{2}$ à partir du malate dans les cellules de gaine, tandis que la sensibilité à $\mathrm{NaCl}$ chez $P$. violaceum serait liée à un ralentissement de décarboxylation du malate dans les gaines.

Reçu le 6 novembre 1985. Accepté le 7 avril 1986.

\section{REMERCIEMENTS}

Nous tenons à remercier Mr le Professeur A. Movse pour la lecture critique du manuscrit, Mr le Dr J. VIDAL qui a bien voulu nous fournir les anticorps et nous initier aux techniques d'immunologie, Melle F. MAURY pour la participation à la présentation du manuscrit.

\section{RÉFÉRENCES BIBLIOGRAPHIQUES}

Aslam M., Huffaker R. C., Williams R. D., 1984. Early effects of salinity on nitrate assimilation in barley seedlings. Plant Physiol., 76, 321-325.

Blondon F., 1968. Contribution à l'étude du développement des graminées fourragères : Ray-grass et dactyle. Thèse Doct. Etat, Sc. Nat., Univ. de Paris, CNRS N ${ }^{\circ}$ AO 2522, 74 p.

Brownell P. F., Crossland C. J., 1972. The requirement for sodium as a micronutrient by species having the $\mathrm{C}_{4}$ dicarboxylic photosynthetic pathway. Plant Physiol., 49, 794-797.

Collins P. D., Hague D. R., 1983. Light-stimulated synthesis of NADP malic enzyme in leaves of maize. J. Biol. Chem., 258, 40124037.

Davies D. D., 1979. The central role of phosphoenolpyruvate in metabolism. Annu. Rev. Plant Physiol., 30, 131-158.

Diallo N., Queiroz-Claret C., 1983. Effet du chlorure de sodium sur le métabolisme carboné de Cakile maritima et Kalanchoe blossfeldiana. Physiol. Vég., 21, 621-632.

Ferron F., Coudret A., Gaudillère J. P., 1977. Etude de la salinité du milieu de culture sur les voies de carboxylation d'une halophyte (Plantago maritima L. var. graminaea) et d'une glycophyte (Plantago lanceolata L.) C. R. Acad. Sci., Paris, 285, Série D, 323-326.

Greenway H., Munns R., 1980. Mechanisms of salt tolerance in nonhalophytes. Annu. Rev. Plant Physiol, 31, 149-190.

Greenway H., Osmond.C. B., 1972. Salt responses of enzymes from species differing in salt tolerance. Plant Physiol., 49, 256-259.

Greenway H., Sims A. P., 1974. Effects of high concentrations of $\mathrm{KCl}$ and $\mathrm{NaCl}$ on responses of malate dehydrogenase (decarboxylating) to malate and various inhibitors. Austr. J. Plant Physiol., 1, 15-29.
Guern J., Mathieu Y., Kurdjian A., 1983. Phosphoenolpyruvate carboxylase activity and the regulation of intracellular $\mathrm{pH}$ in plant cells. Phvsiol. Vég., 21, 855-866.

Hagues D. R., Sims T. L., 1980. Evidence for light-stimulated synthesis of PEPC in leaves of maize. Plant Physiol., 66, 506-509.

Hamza M., 1977. Action de différents régimes d'apport de chlorure de sodium sur la physiologie de deux légumineuses : Phaseolus vulgaris (sensible) et Hedysarum carnosum (tolérante). Relations hydriques et relations ioniques. Thèse Doct. Etat, Université Paris VII, $251 \mathrm{p}$.

Hatch M. D., 1969. Light-induced changes in the content of some enzymes of the $\mathrm{C}_{4}$ dicarboxylic acid pathway of photosynthesis and its effect on the characteristic of photosynthesis. Phytochemistry, 8, 697-706.

Hatch M. D., Slack C. R., 1970. Photosynthetic $\mathrm{CO}_{2}$ fixation pathways. Annu. Rev. Plant Physiol., 21, 141-162.

Kahane I., Poljakoff-Mayber A., 1968. Effect of substrate salinity on the activity for protein synthesis in pea roots. Plant Physiol., $\mathbf{4 3}$. $1115-1119$

Lane M. D., Maquyama H., Easterday R. L., 1969. Phosphoenolpyruvate carboxylase from peanut cotyledon. Method. Enzymol., 13, 277-283.

Lavergne D., Bismuth E., Champigny M. L., 1979. Physiological studies of two cultivars of Pennisetum : P. americanum $23 \mathrm{DB}$, a cultivated species and P. mollissimum, a wild species. Z. Pflanzenphysiol., 91, 291-303.

Levitt J., 1972. Responses of plants to environmental stresses. Academic Press, New York, London, 697 p. 
Lowry O. H., Rosebrough N. J., Farr A. L., Randall R. J., 1951 Protein measurement with the Folin phenol reagent. J. Biol. Chem. 193, 265-275.

Meiri A., Poljakoff-Mayber A., 1970. Effect of various salinity regimes on growth, leaf expansion and transpiration rate of bean plants. Soil Sci., 109, 26-34.

Miginiac-Maslow M., Vidal J., Bismuth E., Hoarau A., Champigny M. L., 1983. Effet de la carence et de la réalimentation en phosphate sur l'équilibre énergétique et l'activité phosphoénolpyruvate carboxylase de jeunes plantes de blé. Physiol. Vég., 21, 325-336.

Osmond C. B., Greenway H., 1972. Salt response of carboxylation enzymes from species differing in salt tolerance. Plant Physiol., 49, 260-263.
Osmond C. B., Laties G. G., 1969. Compartmentation of malate in relation to ion absorption in beet. Plant Physiol., 44, 7-14.

Ouchterlony O., 1958. Diffusion in gel methods for immunological analysis. Prog. Allergy, 5, 1-78.

Siegel S. M., Siegel B. Z., Massey J., Lahne P., Chen J., 1980. Growth of corn in saline waters. Physiol. Plant., 50, 71-73.

Van Steveninck R. F. M., Steveninck M. E., Stelzer R., Laüchli A. 1982. Studies on the distribution of $\mathrm{Na}$ and $\mathrm{Cl}$ in two species of lupin (Lupinus luteus and Lupinus angustifolius) differing in salt tolerance. Physiol. Plant., 56, 465-473.

Vidal J., Gadal P., 1983. Influence of light on phosphoenolpyruvate carboxylase in sorghum leaves. II. Immunochemical study. Physiol. Plant., 57, 124-128. 